\title{
ENFERMAGEM NO CENTRO DE MATERIAL ESTERILIZADO - A PRÁTICA DA EDUCAÇÃO CONTINUADA ${ }^{1}$
}

\author{
Mara Cristina Bicudo de Souza ${ }^{2}$ \\ Maria Isabel Pedreira de Freitas Ceribelli ${ }^{3}$
}

Souza MCB, Ceribelli MIPF. Enfermagem no centro de material esterilizado - a prática da educação continuada. Rev Latino-am Enfermagem 2004 setembro-outubro; 12(5):767-74.

Esta pesquisa tem por objetivo caracterizar a prática da Educação Continuada (EC) oferecida ao pessoal que desenvolve atividades de enfermagem nos Centros de Material Esterilizado de hospitais da microrregião de São José dos Campos, SP. É uma pesquisa descritiva, com análise quantitativa, realizada com enfermeiros, técnicos e auxiliares de enfermagem, auxiliares de esterilização e estudantes de enfermagem, por meio de entrevista estruturada. Verificou-se que $31,2 \%$ dos entrevistados (um enfermeiro e dezenove funcionários) participaram de EC; $64,5 \%$ dos funcionários não foram motivados a participar. A EC é do tipo teórico-prática com supervisão direta e de caráter opcional; a avaliação é realizada por meio da observação e análise do desempenho do funcionário, sem um instrumento formal. Observou-se, ainda, a necessidade de um efetivo serviço de EC com programas estruturados.

DESCRITORES: educação continuada em enfermagem; recursos humanos de enfermagem

\section{NURSING AT THE STERILIZED MATERIAL CENTER - CONTINUING EDUCATION PRACTICE}

This research aims to characterize Continuing Education (CE) practices offered to the staff involved in nursing activities at Sterilized Material Centers of hospitals located in the micro-region of São Jose dos Campos, Brazil. A descriptive research with quantitative analysis was carried out through structured interviews, with nurses, nursing technicians and auxiliaries, sterilization assistants and nursing students. It was found that $31.2 \%$ of the interviewees (one nurse and nineteen employees) took part in CE; $65.4 \%$ of the employees were not motivated to participate. The CE is theoretic-practical and optional, with direct supervision; employees are evaluated through performance observation and analysis, without any other formal instruments. We also observed the need for an actual CE service of CE with structured programs.

DESCRIPTORS: continuing nursing education; nursing staff

\section{ENFERMERÍA EN EL CENTRO DE MATERIAL ESTERILIZADO - LA PRÁCTICA DE LA EDUCACIÓN CONTINUA}

La finalidad de esta investigación es caracterizar la práctica de la Educación Continua (EC) ofrecida al personal que desarrolla actividades de enfermería en los centros de material esterilizado de los hospitales de la micro-región de São Jose dos Campos. Esta es una investigación descriptiva con análisis cuantitativo, realizada por medio de entrevistas estructuradas con enfermeras, técnicos y auxiliares de enfermería, auxiliares de esterilización y estudiantes de enfermería. Fue encontrado que el $31.2 \%$ de los entrevistados (un enfermero y diecinueve empleados) participaron en la EC; $y$ 65.4\% de los empleados no fueron motivados a participar. La EC es del tipo práctico-teórico con supervisión directa y de carácter opcional; los empleados son evaluados por medio de la observación y análisis de su desempeño, sin ningún otro instrumento formal. También se observó la necesidad de un efectivo servicio de EC con programas estructurados.

DESCRIPTORES: educación continua en enfermería; personal de enfermería

\footnotetext{
${ }^{1}$ Trabalho extraído da dissertação de mestrado apresentada a Universidade Estadual de Campinas, $2001 ;{ }^{2}$ Enfermeira, Mestre, Professor Assistente III da Universidade de Taubaté, e-mail: marajac@uol.com.br; ${ }^{3}$ Enfermeira, Doutor, Professor da Universidade Estadual de Campinas
} 
INTRODUÇÃO

$\boldsymbol{A}$ Educação Continuada (EC) é uma das formas de se proporcionar o desenvolvimento e o aperfeiçoamento dos recursos humanos das instituições.

$\mathrm{Na}$ área de saúde observa-se, também, a necessidade de educar os profissionais de enfermagem para que se ofereça melhor assistência ao paciente que depende dos serviços da organização hospitalar. A enfermagem é uma profissão que requer constante atualização, devido à evolução tecnológica e científica. Nesse sentido, a enfermagem utiliza, muitas vezes, o serviço de EC para oferecer aos seus funcionários conhecimentos para uma atuação eficaz.

O Centro de Material Esterilizado (CME) é parte fundamental do contexto hospitalar, é o local responsável pelo expurgo, preparo, esterilização e distribuição dos materiais e equipamentos usados no centro cirúrgico e demais unidades de um hospital. Sua grande importância deve-se às atividades nele desenvolvidas, envolvendo ações de terceiros, médicos e outros profissionais de enfermagem, em procedimentos críticos e semicríticos junto aos pacientes.

Considerado uma unidade vital, o CME necessita de funcionários preparados adequadamente para cada área e funções que assumam. Os administradores dos hospitais devem estar conscientes dessa necessidade, dando, portanto, maior atenção a esses profissionais, os quais, embora não estejam prestando assistência direta ao paciente, executam atividades extremamente importantes (assistência indireta).

Para se obter êxito no funcionamento do CME, é necessário que os funcionários sejam em quantidade e qualidade adequadas e que exista um efetivo programa de EC, visando ajudar o funcionário a manter-se atualizado, a trabalhar com conhecimento e competência, bem como a desenvolver habilidade para analisar problemas e trabalhar em equipe ${ }^{(1)}$, "...mais preparados para lidar com as mudanças que vão se dando no setor e no contexto mais geral, compreendendo melhor a si próprio, a sua realidade, fazendo parte da construção de um projeto coletivo de sociedade igualitária, solidária e justa"(2).

A importância da $E C$ como fator de mudanças é relatada desde 1974, e a Assembléia Mundial de Saúde já alertava sobre a necessidade de se desenvolver sistemas nacionais de EC para os profissionais da saúde ${ }^{(3)}$. Pode-se afirmar que: "Apesar de o conceito de educação continuada não ser novo, persiste muito variado; não significa a mesma coisa para todas as pessoas e não é definido da mesma maneira pelos diferentes grupos de saúde. ... às vezes, é considerada como treinamento periódico de 'refrescamento' para manter o profissional atualizado em seus conhecimentos" ${ }^{\prime(4)}$.

A EC é um dos caminhos para uma assistência de qualidade, respeitando-se o paciente e o profissional. Engloba programas de ensino que proporcionam aos trabalhadores oportunidades de aprendizagem e desenvolvimento de habilidades em suas ações profissionais, "... integrando o processo produtivo ao educativo, contemplando as necessidades da instituição, mas também as necessidades, as expectativas de elaboração de conhecimentos, experiências que vão além das exigências profissionais imediatas, respeitando as particularidades pessoais" ${ }^{\text {"(2) }}$. Estudos realizados mostram que as tarefas executadas pelos funcionários no CME são divididas nas diversas áreas e fragmentadas de acordo com as rotinas estabelecidas pela organização. Cabe ressaltar a importância do enfermeiro como gerenciador dessas atividades, sendo responsável pela orientação e pelo desenvolvimento profissional dos funcionários que trabalham no CME, oferecendo-Ihes condições para que possam alcançar os objetivos propostos. Portanto, deve o enfermeiro estar à frente dos programas de $E C^{(5-6)}$.

Na prática da enfermagem, o enfermeiro é um educador em todos os campos de sua atuação. Estará sempre comprometido com a função de educar, pois sua prática envolve ações na área social, no ensino, na pesquisa, na administração, na responsabilidade legal e na participação em associações de classe ${ }^{(7)}$.

O profissional que trabalha no CME precisa desenvolver habilidades e maturidade profissional para atender à demanda de trabalho que há no dia-a-dia da unidade. Necessita sentir-se valorizado e competente em suas tarefas e, dessa forma, alcançar satisfação profissional e social.

Com base na experiência profissional, na atuação e observação constante do serviço realizado no $\mathrm{CME}$, denota-se a necessidade de práticas educativas de enfermagem na área e a pertinência de uma pesquisa sobre 0 assunto.

Acredita-se que essa pesquisa contribuirá para o conhecimento das práticas educativas que os hospitais da Microrregião de São José dos Campos,SP oferecem aos trabalhadores que desenvolvem atividades de 
enfermagem no CME e promover, ainda, reflexão sobre possíveis e específicas propostas para modificações.

Teve como objetivo caracterizar a prática da Educação Continuada, oferecida para o pessoal de enfermagem que atua nos Centros de Material Esterilizado de hospitais da Microrregião de São José dos Campos, descrevendo as estratégias de ensino-aprendizagem, bem como as formas de realização, supervisão e avaliação.

\section{MÉTODO}

Este estudo é de natureza descritiva e exploratória. O projeto foi aprovado pelo Comitê de Ética em Pesquisa da Universidade de Taubaté, sendo a pesquisa realizada na Região do Vale do Paraíba Paulista (RVPP) Estado de São Paulo, geograficamente dividida em seis microrregiões. Localiza-se no eixo Rio de Janeiro-São Paulo, é cortada pela rodovia Presidente Dutra e está a leste, no Estado de São Paulo, entre as serras do Mar e da Mantiqueira ${ }^{(8)}$.

A microrregião de São José dos Campos compreende oito municípios. Foi selecionada como campo de estudo por concentrar o maior número de hospitais da região e por ser a microrregião de maior concentração demográfica - 1830,03 habitantes $/ \mathrm{km}^{2(9)}$.

Para o estudo foram selecionados todos os enfermeiros, técnicos de enfermagem, auxiliares de enfermagem, auxiliares de esterilização (antigos atendentes de enfermagem) e estudantes de enfermagem (pessoas sem formação na área de saúde e que atualmente fazem curso de enfermagem - estágio extracurricular), os quais compõem a equipe que trabalha nos hospitais da microrregião de São José dos Campos e que tinham o profissional enfermeiro como responsável pelo CME ou pelo CME e Centro Cirúrgico (CC).

A pesquisa foi realizada em nove hospitais, sendo dois $(22,2 \%)$ de grande porte (151 a 500 leitos), cinco $(55,6 \%)$ de médio porte (51 a 150 leitos) e dois $(22,2 \%)$ de pequeno porte (até 50 leitos), quatro (44,5\%) particulares, dois $(22,2 \%)$ municipais, dois $(22,2 \%)$ filantrópicos e uma fundação. A maioria, sete $(77,8 \%)$, é composta de hospitais gerais.

Foram entrevistados 64 sujeitos: nove enfermeiros, dois técnicos de enfermagem, 28 auxiliares de enfermagem, 23 auxiliares de esterilização e dois estudantes.

Para a coleta de dados junto aos sujeitos da pesquisa, foram realizadas entrevistas seguindo formulários de forma a atender às categorias dos respondentes, após anuência dos mesmos, mediante a assinatura do Termo de Consentimento da Instituição pelo administrador, e do Termo de Consentimento Livre e Esclarecido de cada participante do estudo. As entrevistas foram conduzidas pessoalmente por uma das pesquisadoras que anotava as respostas e as apresentava, no final, ao entrevistado para ciência dos registros efetuados e para a sua aprovação. Foram realizadas no período de 8 de janeiro a 8 de fevereiro de 2001 .

Antes das entrevistas, os formulários foram avaliados por meio de um pré-teste, aplicado em populações com as mesmas características daquelas do estudo, pertencentes à Microrregião de Caraguatatuba.

Os dados foram tabulados eletronicamente, com auxílio do programa Microsoft Excel - 97, sob orientação do Serviço de Estatística da Comissão de Pesquisa da Faculdade de Ciências Médicas-UNICAMP. O programa de computador utilizado para análise estatística foi o $S A S$ System for Windows (Statistical Analysis System) versão 6.12. SAS Institute, 1989-1996, NC, USA.

\section{RESULTADOS E DISCUSSÃO}

As atividades de $\mathrm{EC}$ oferecidas aos enfermeiros e funcionários* dos CMEs serão apresentadas e analisadas, de forma conjunta de acordo com as respostas obtidas.

Pode-se verificar que apenas um enfermeiro, dentre os nove entrevistados, relatou ter recebido EC prévia**, para sua atuação no CME, por meio de visitas a outros hospitais. Portanto, não será mais abordada a EC oferecida a esse profissional, por ter sido citada por somente um deles. Os administradores, geralmente, contratam o enfermeiro para gerenciar o centro cirúrgico e estendem sua responsabilidade ao $\mathrm{CME}$. Parece não existir uma valorização, tanto por parte desses, como dos profissionais de enfermagem da importância e autonomia de ação dos enfermeiros no CME. Segundo alguns autores, visitas são tipos informais de EC que devem ser

\footnotetext{
* Técnicos de enfermagem, auxiliares de enfermagem, auxiliares de esterilização e estudantes de enfermagem

** Treinamento ou preparo inicial para realizar uma determinada função
} 
consideradas como atividades educativas ${ }^{(10-11)}$.

Dos 55 funcionários, $19(34,6 \%)$ relataram ter, em algum momento, participado de atividades de EC no CME, embora não tenham especificado, quando solicitado, quais as atividades que lhe foram oferecidas.

Constatou-se, também, nas respostas, que, dos 19 funcionários, $16(84,2 \%)$ tiveram EC prévia, e três $(15,8 \%)$ deles não continuaram recebendo EC. No entanto, dos 16 que tiveram EC, $13(81,2 \%)$ continuam recebendo atualização. E outros três (18,8\%) que não tiveram EC prévia, atualmente recebem EC para atuar no CME.

A continuidade da EC tem dois aspectos importantes: 1) a sociedade em constante mudança leva o homem a enfrentar novos desafios. Torna-se necessária uma nova ação frente à evolução dessa sociedade que Ihe apresenta tarefas que exigem desenvolvimento de outras capacidades de ação e trabalho; 2) a força de trabalho do homem, ao acompanhar a evolução social, gera mudanças. À medida que o homem modifica seu grau de desenvolvimento, aumenta sua necessidade de mais educação ${ }^{(10)}$.

Quanto à análise das atividades de EC que os enfermeiros desenvolvem com seus funcionários, cinco $(55,6 \%)$ relataram como atividade a orientação no dia-adia e três $(33,3 \%)$ citaram como sendo "outras" atividades, porém, não as especificaram.

Somente um enfermeiro relatou não desenvolver atividade de EC com seus funcionários.

Quando os enfermeiros foram questionados se consideravam importantes a EC para os funcionários do CME e o porquê dessa consideração, os nove (100\%) enfermeiros responderam de modo afirmativo e justificaramna como um processo de atualização e desenvolvimento pessoal.

Observou-se que cinco (55,6\%) enfermeiros relataram motivar seus funcionários para a EC por meio de orientações sobre a importância da mesma, e que um enfermeiro mencionou realizar acordo de horas, isto é, o funcionário desconta as horas despendidas com atividades de EC em folga, ou recebe como hora extra, o que pode ser considerado como recurso de motivação. No entanto, observou-se, ainda, que três $(33,3 \%)$ relataram não motivarem seus funcionários para a EC.

O funcionário, quando motivado, desenvolve suas atividades de maneira equilibrada e produtiva. Cabe ao enfermeiro responsável pelo CME estimular e orientar sua equipe quanto à importância do trabalho que realiza, levando-a a reconhecer a utilidade de seus procedimentos técnicos para o hospital e a necessidade de atualização. Assim, os funcionários sentem-se valorizados e dispostos para o trabalho ${ }^{(12)}$. Os valores da profissão fundamentam a ação gerencial do enfermeiro que, no exercício de suas atividades, deve estar atualizado em seus conhecimentos e assim conduzir sua equipe e sua prática em uma assistência de qualidade ao ser humano ${ }^{(13)}$.

Quanto aos funcionários, 11 (20\%) confirmaram receber estímulo dos enfermeiros, por meio de orientações sobre a importância de estar sempre aprendendo e renovando os conhecimentos no CME. Quatro (7,3\%) funcionários revelaram ter interesse próprio, embora não recebam estímulo, e 36 (65,4\%) disseram "prefiro não responder à questão". Diante da recusa pela maioria dos funcionários, percebe-se que não há uma interação enfermeiro/funcionários que evidencie condições de estímulo. O conjunto de condições comunicativas, teóricas, sociais e intencionais é que direcionam a conduta do participantes mediante atitudes, idéias e sentidos ${ }^{(14)}$.

$\mathrm{Na}$ EC o aspecto "querer aprender", que deve estar sempre presente, é importante e, quando em estado latente, deve ser estimulado ${ }^{(15)}$.

A maioria dos enfermeiros (75\%) que desenvolve EC e $89,4 \%$ dos funcionários que a recebem, relataram ser a EC, no CME, abrangente nas diversas áreas, em contraposição à minoria, 12,5\% dos enfermeiros e 5,3\% dos funcionários, que responderam ser específica para as áreas de expurgo, de preparo e acondicionamento, de esterilização e de guarda e distribuição de materiais.

Autores relatam ser uma medida importante que o funcionário adquira/desenvolva conhecimento e habilidade referentes às diversas atividades executadas no $\mathrm{CME}$, pois, quando da necessidade de substituir pessoal em qualquer uma das áreas, isso não causará insatisfações ou diminuição do rendimento do trabalho ${ }^{(6)}$.

A EC oferecida é predominantemente teóricoprática, segundo o relato de $42,1 \%$ dos funcionários. "No ensino, teoria e prática devem informar-se uma à outra, pois se interpenetram. A prática, por exemplo, deve informar a teoria e solicitar-lhe, ao mesmo tempo, os fundamentos que a alicerçam"(14).

No CME, as atividades técnicas (habilidades práticas) necessitam de embasamento científico (fundamentação teórica), para serem realizadas. O acondicionamento de roupas, materiais e instrumentais, entre outras atividades, estão fundamentados em 
conceitos tecnológicos e de controle de infecção hospitalar. Não é ao acaso que, numa caixa cirúrgica, a disposição dos materiais seguem uma seqüência: os instrumentos mais pesados são colocados na parte inferior e as pinças são agrupadas de acordo com seus tipos e utilização na cirurgia. Também há seqüência de disposição, na mesa de instrumentação. As dobras de aventais e campos cirúrgicos são esquematizados para pronto uso e sem desperdício de tempo e movimentos pela equipe; há o risco de contaminação quando são preparados de forma incorreta, ou mesmo quando do seu manuseio.

Diante desses aspectos, associar a teoria à prática proporcionará, ao funcionário, melhor assimilação e um raciocínio lógico sobre a necessidade e importância dos pequenos cuidados com os materiais.

Orientações teóricas, sem demonstrações práticas, também são utilizadas no setor, por exemplo, conceito sobre: autocuidado, quando do manuseio de materiais contaminados, composição de agentes químicos e efeitos nos indivíduos, condutas posturais, forma de colocação dos materiais nas máquinas de esterilização, para a atuação dos agentes esterilizantes e circulação nas áreas do CME, entre outras.

A EC teórica, realizada em sala de aula, fornece conceitos para a abstração de conhecimentos que, muitas das vezes, por não serem vivenciados pelo aprendiz, dificulta sua compreensão e o entendimento da lógica implícita, nesse conhecimento, para a construção de capacidade crítica em sua ação ${ }^{(14)}$.

As atividades práticas, quando desenvolvidas isoladamente, tornam os funcionários meros executores de tarefas, responsáveis por produção. Apesar do processo ensino-aprendizagem prever articulação teórico-prática, há situações em que se restringe a um fazer mecânico, não reflexivo.

A didática escolanovista, ao valorizar as experiências, aceita o emprego da exposição oral e da demonstração. A aula prática em laboratórios ou no próprio local de trabalho é classificada como demonstração didática operacional que se baseia em técnica de trabalho, com auxílio, ou não, de instrumentos, máquinas e equipamentos. Essa forma de ensinar torna o trabalho mecanicista, valorizando o que é programado e desejado, a eficiência é muito mais valorizada do que o espírito crítico e de criatividade ${ }^{(16)}$

"A unidade entre a teoria e a prática, é assegurada pela simultaneidade e reciprocidade, pela autonomia e dependência de uma em relação à outra. É necessário, no entanto, compreender essa relação como processo por meio do qual se constrói o conhecimento"(16).

Pode acontecer de o funcionário falhar em um procedimento, quando do preparo do material, e não the dar a atenção necessária, ou seja, não tornar a executálo, por achar que um pequeno detalhe não altera a finalidade do produto, ou mesmo o produto, final. Portanto, o conhecimento técnico-científico do trabalho que realiza o ajuda a realizá-lo com qualidade.

Os responsáveis pela EC não devem somente dar um saber sistematizado aos funcionários. Esses devem vivenciar e refletir juntos, despertar no funcionário a vontade de aprender. O saber gerado na prática cotidiana possibilita maior apreensão e torna o aprendizado efetivo. Deve haver um diagnóstico das necessidades e dos programas que deverão ser propostos, levando os funcionários a se sentirem motivados para aprender ${ }^{(11)}$.

A EC foi realizada sem supervisão, para 10,5\% dos funcionários, ou seja, sem a permanência de um enfermeiro no local de trabalho coordenando as atividades do CME, e com supervisão para $89,5 \%$ deles. Dos que foram supervisionados, $76,5 \%$ tiveram supervisão direta e $23,5 \%$, supervisão indireta.

No CME, as atividades realizadas, embora de forma indireta, estão relacionadas com o cuidado aos pacientes, devendo, portanto, também ser supervisionadas pelo enfermeiro. À medida que os funcionários recebem EC, o enfermeiro deve supervisionar o desenvolvimento das atividades, o que permitirá retroalimentação do diagnóstico da EC oferecida.

Toda atividade de enfermagem executada pelos funcionários no hospital deve estar diretamente sob supervisão de enfermeiros, cujo papel é de orientador e facilitador no ambiente de trabalho. Essa função tem caráter educativo, podendo ser realizada por meio de observação direta, análise de registro, entrevista, reunião e discussão em grupo, entre outras ${ }^{(17)}$.

A adoção de supervisão direta permite que o enfermeiro acompanhe cada funcionário, observando o seu desempenho. A supervisão indireta poderá ser empregada no grupo; no entanto, por ser geral, o enfermeiro pode deixar de perceber pontos importantes que necessitem intervenção educacional.

Constatou-se, pelas respostas, que o caráter da EC oferecida aos funcionários, segundo o relato de 11 $(57,9 \%)$ dos 19 funcionários, é opcional, e oito $(42,1 \%)$ 
relataram que foram obrigados a participar da mesma.

A EC deve ser planejada para os funcionários, considerando-se a cultura organizacional como uma estratégia para proporcionar-lhes condições de trabalho seguro, desenvolver seus conhecimentos e adaptá-los ao ambiente de trabalho. Entende-se que a opção para participar de atividades de desenvolvimento pessoal advém de um processo de esclarecimento ao funcionário, com estímulos suficientes para levá-lo a procurar meios de crescimento e de valorização naquilo que lhe é dado para executar.

Quando questionados sobre a realização de uma avaliação, dos oito $(88,9 \%)$ enfermeiros que realizaram EC, cinco $(62,5 \%)$ responderam que não fazem avaliação, e três $(37,5 \%)$ que a realizam após oferecer EC.

Somente quatro $(21 \%)$ dos funcionários não são avaliados quando da EC e os demais relataram que recebem algum tipo de avaliação, predominando a avaliação das atividades no dia-a-dia, com observação e análise do desempenho do funcionário na função que executa para $73,7 \%$. Observou-se que no CME a significância, aos olhos dos enfermeiros e dos funcionários sobre avaliação, assume concepções diferentes. A maioria dos enfermeiros ainda não reconhecem como avaliação o espaço interativo do ambiente de trabalho. No entanto, os funcionários consideraram-se avaliados pela regulação e monitoramento de suas atividades.

Toda avaliação envolve conhecimento, podendo ser realizada por meio de testes objetivos, testes de respostas abertas ou questões dissertativas; habilidades, por meio de exercícios práticos, os quais são os instrumentos essenciais para se avaliar as habilidades assimiladas e desenvolvidas em um treinamento; experiências do dia-adia, por meio de observação e análise do desempenho do funcionário no cargo (avaliação de desempenho) e por atitudes, em que há observação direta em uma sala de aula e no trabalho ${ }^{(18)}$.

Quanto à freqüência de realização da EC, observou-se que a maior parte, $68,5 \%$ dos funcionários, relatou recebê-la sempre que é adquirido um novo equipamento; 10,5\% informaram ser mensal; 10,5\% mencionaram ser somente na admissão; e 10,5\% não informaram.

A experiência no CME tem mostrado que a maioria dos enfermeiros reúne-se com seus funcionários para orientá-los, geralmente, quando são adquiridos novos equipamentos pela instituição, o que corrobora com os dados obtidos. Percebe-se, também, que ocorre quando se pretende demonstrar a mudança de agentes químicos, a montagem de uma nova caixa cirúrgica e materiais consignados para cirurgias especializadas. Os tipos de programas de EC variam de acordo com o enfermeiro e com o que a instituição pretende oferecer a seus funcionários.

A EC engloba vários tipos de programas, podendo ser: de orientação inicial, visando a adaptação do funcionário, por meio de normas, rotinas, e procedimentos de uma determinada instituição - facilita a integração do funcionário; de treinamento, que visa preparar o funcionário para melhor assumir um cargo ou função - admissão ou novas funções; e de atualização - os funcionários recebem informações para melhor ampliar seus conhecimentos e habilidades no seu campo específico de atuação, incluindo curso intra e extra-instituição, e pode constituir-se de visitas, estágios e participação em eventos promovidos por outras instituições ${ }^{(11)}$.

O treinamento é considerado como um processo educacional, de curto prazo, que envolve transmissão de conhecimentos específicos relacionados ao trabalho, atitudes diante de aspectos organizacionais, de tarefas e do ambiente, bem como o desenvolvimento de habilidades ${ }^{(19)}$.

Na população estudada, observou-se que, pela freqüência da EC oferecida, parece tratar-se de um programa de treinamento. Aos funcionários são transmitidas informações tecnológicas e relacionadas à admissão, caracterizando assim as subáreas do processo de treinamento. Com relação à tecnologia, pode-se entender também como um processo de atualização, ou como um treinamento técnico para capacitar o ser humano a trabalhar com a máquina. No entanto, esse não seria um objetivo suficiente, faz-se necessário preparar os funcionários para trabalharem uns com os outros e pelo paciente, saber criar, se posicionar, refletir e agir “... recriar todo o processo educativo, mais instrumentalizado, transformado, para dar conta de um trabalho que não é simples transferência de informação aos indivíduos..."(20).

Quanto à responsabilidade pela $\mathrm{EC}$, essa tem sido do enfermeiro, citado como "seu chefe imediato", segundo o relato de 11 (57,9\%) dos funcionários. Observa-se, também, que foi delegada ao "colega de trabalho" em dois $(10,5 \%)$ casos. O Serviço de EC foi mencionado apenas uma vez $(5,3 \%)$.

Toda a coordenação e a execução de um programa 
de desenvolvimento de recursos humanos, estabelecido por um serviço de EC, ou não, devem ser exercidas pela enfermeira da unidade de trabalho. As autoras relatam ainda que os funcionários que trabalham na unidade são considerados instrutores em potencial e que, quando é delegado a um funcionário orientar seu colega, o enfermeiro deve prepará-lo, orientando-o primeiramente para esse papel $^{(10)}$.

Quando há um Serviço de EC, cabe à enfermeira responsável por ele a tarefa de realizar o treinamento de todo o pessoal de enfermagem no que tange à parte teórica. A parte prática deve ser realizada pelas enfermeiras da unidade e coordenada pela EC ${ }^{(21)}$.

Quanto ao horário em que foi realizada a EC, foi citado pela maioria, $18(94,7 \%)$ funcionários, como sendo no período do turno de trabalho.

Percebe-se, na prática profissional, que as instituições, na maioria das vezes, oferecem a EC no período do turno de trabalho, para facilitar a participação dos funcionários que, fora desse horário, precisam de remuneração extra, além de os mesmos não disporem de tempo para retornarem à instituição para esse tipo de atividade. Acrescenta-se ainda o fato de ser a enfermagem exercida predominantemente por mulheres que têm também ocupações domésticas, as quais podem agir como fator impeditivo para o retorno ao local de trabalho.

Quando o funcionário é remunerado para essas atividades, o aspecto financeiro pode agir como fator de estímulo ou, por outro lado, ser fator de não realização pela instituição, por considerá-las um investimento oneroso.

Percebe-se que "quando se pretende desenvolver um processo educativo junto a adultos no seu trabalho, ou para o seu trabalho, deve-se levar em consideração que o ponto de partida da aprendizagem é a experiência adquirida em sua vida diária e que cada um já vem com uma bagagem específica”, ${ }^{\text {(20) }}$. Ao enfermeiro, na posição de educador, caberá proporcionar meios para que aconteça o aprendizado, dando sempre ênfase ao programa de EC

\section{REFERÊNCIAS BIBLIOGRÁFICAS}

1. Salzano SDT, Silva A, Watanabe E. O Trabalho do Enfermeiro no Centro de Material. Rev Paul Enfermagem 1990 setembro/ dezembro; 9(3):103-8.

2. Bagnato MHS. Educação continuada na área de saúde: uma aproximação crítica. In: Bagnato MHS, Cocco IM, Sordi $\mathrm{MRL}$, organizadores. Educação, saúde e trabalho: antigos problemas, novos contextos outros olhares. Campinas (SP): Alíneas; 1999. com comunicação efetiva, objetivos bem definidos e processos de avaliação, buscando motivar seus funcionários a interessar-se pelo que é abordado, para que possam aprender.

\section{CONCLUSÕES}

O estudo permitiu concluir que dos 64 entrevistados, somente $31,2 \%$ participaram, em algum momento, das atividades de EC em CME. Essa envolveu um enfermeiro e 19 funcionários.

Dos nove enfermeiros, oito oferecem EC aos funcionários, e essa consistiu em orientações realizadas no dia-a-dia. Dos funcionários participantes, $65,4 \%$ não responderam sobre a utilização de estímulos que motivassem sua participação na $E C$, o que pode sugerir não se sentirem estimulados para tal. A freqüência de oferta da EC é por ocasião da chegada de um novo equipamento, portanto, com realização de programas de treinamento, os quais, inseridos como EC, permitem aos funcionários o desenvolvimento das atividades com fundamentação nos conhecimentos.

A EC é do tipo teórico/prática com supervisão direta e de caráter opcional. Sua avaliação utiliza observação e análise, pelo enfermeiro, do desempenho do funcionário na função que executa, sem instrumento formal de verificação.

Face a essas conclusões cumpre, ainda, comentar sobre a necessidade de uma análise do contexto dos CMEs estudados, pelos responsáveis, e a adoção de um efetivo serviço de EC, com programas estruturados que possibilitem a continuidade do desenvolvimento dos recursos humanos envolvidos nas atividades do setor. Sugere-se ainda a elaboração de um protocolo para a EC e a adoção de uma metodologia participativa que possibilitem aos funcionários a construção pessoal de significados, integrando-os ao sistema institucional de maneira ativa e participativa.

3. OguissoT. A educação continuada como fator de mudanças: visão mundial. Rev Tec Enf Nursing 2000 janeiro; 20(1):22-9. 4. Bastos NCB. A Educação Contínua nas Profissões de Saúde. Rev Fund SESP 1978; 23(2):31-7.

5. Moura MLP. Gerenciamento da Central de Material e Esterilização para Enfermeiros: Fundamentos Teóricos, Organizacionais e Estruturais. São Paulo (SP): SENAC; 1996. 6. Silva MDA, Rodrigues AL, Cesareti IUR, organizadores. Enfermagem na Unidade de Centro Cirúrgico. São Paulo (SP): EPU; 1997. 
7. Crivari MMF, Silva ML. Educação em Serviço - Treinamento para alunos do 4ํano de Enfermagem. Rev Bras Enfermagem 1996 janeiro/março; 48(1):65-74.

8. Instituto Brasileiro de Geografia e Estatística. (BR). Censo Demográfico: resultados preliminares. Relação da Divisão Territorial do Brasil com indicação das Unidades da Federação, Mesorregiões Homogêneas, Microrregiões Homogêneas e Municípios com respectivas subordinações. Rio de Janeiro (RJ): IBGE; 1992.

9. Instituto Brasileiro de Geografia e Estatística. (BR). Censo Demográfico: dados parciais. São Paulo (SP): IBGE; 2000.

10. Silva MJP, Pereira LL, Benko MA, organizadores. Educação Continuada: estratégica para o desenvolvimento do pessoal de enfermagem. Rio de Janeiro (RJ): Marques Saraiva; 1989. 11. Leite MMJ, Pereira LL. Educação Continuada em Enfermagem. In: Kurcgant P, organizador. Administração em Enfermagem. São Paulo (SP): EPU; 1991. p. 147-63.

12. Silva MVG. A Equipe de Enfermagem do Centro de Material: um grupo esquecido. [Dissertação]. Rio de Janeiro (RJ): Escola de Enfermagem Anna Nery/UFRJ; 1995.

13. Trevisan MA, Mendes IAC, Lourenço MR, Shinyashiki GT. Aspectos éticos na ação gerencial do enfermeiro. Rev Latinoam Enfermagem 2002 janeiro-fevereiro; 10(1):85-9.

14. Abud MJM. Profesores de ensino superior: características de qualidade. Taubaté (SP): Cabral Editora Universitaria; 2001. 15. Pinho DLM. Educação Continuada de recursos Humanos na área de Enfermagem: um estudo exploratório. [Dissertação]. Brasília (DF): Faculdade de Educação/UnB; 1994.

16. Veiga IPA. Nos laboratórios e oficinas escolares: a demonstração didática. In: Veiga IPA, organizador. Técnicas de ensino: por que?. Campinas (SP): Papirus; 1995.

17. Cunha K de C. Supervisão em Enfermagem. In: Kurcgant $\mathrm{P}$, organizador. Administração em Enfermagem. São Paulo (SP): EPU; 1991.

18. Carvalho AV, Nascimento LP. Treinamento de Recursos Humanos. In: Kurcgant P, organizador. Administração de Recursos Humanos. São Paulo (SP): Pioneira; 1999.

19. Chiavenato I, organizador. Recursos Humanos. São Paulo (SP): Atlas; 2000.

20. Salum NC, Prado ML. Educação Continuada no trabalho: uma perspectiva de transformação da prática e valorização do trabalhador (a) de enfermagem. Rev Texto e Contexto Enfermagem 2000 maio/agosto; 9(2):298-311.

21. Cavalheiro ERA, Coli RCP. Treinamento em Enfermagem. Rev Paul Enfermagem 1993 maio/agosto; 12(2):57-9. 